NASA Technical Memorandum 79298

\title{
TIME-DEPENDENT DIFFERENCE THEORY FOR NNOISE PROPAGATION \\ IN A TWO-DIMENSIONAL DUCT
}

\section{(NASA-TM-79298)} THEORY FOR NOISE PROPAGATION IN TWO-DIMENSIONAL DUCT (NASA) $13 \mathrm{p}$ HC $\mathrm{B} 02 / \mathrm{MF} A 01$
DIFFERENCE A

CSCI 20A
N80-12822
G3/71 46202

Kenneth J. Baumeister

Lewis Research Center

Cleveland, Ohio

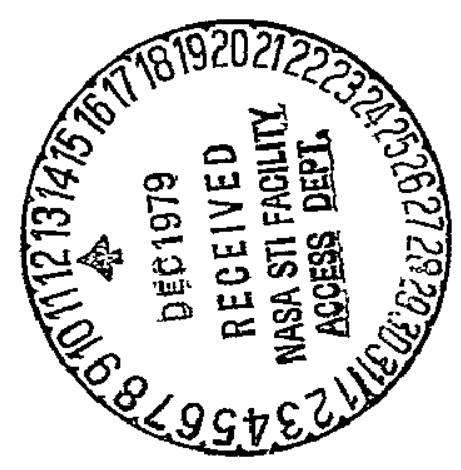

Prepared for the

Eighteenth Aerospace Sciences Meeting

sponsored by the American Institute of Aeronautics and Astronautics

Pasadena, California, January 14-16, 1980 
Kenneth J. Baumeister*

National Aeronautics and Space Administration

Lewis Research Center

Cleveland, Ohio

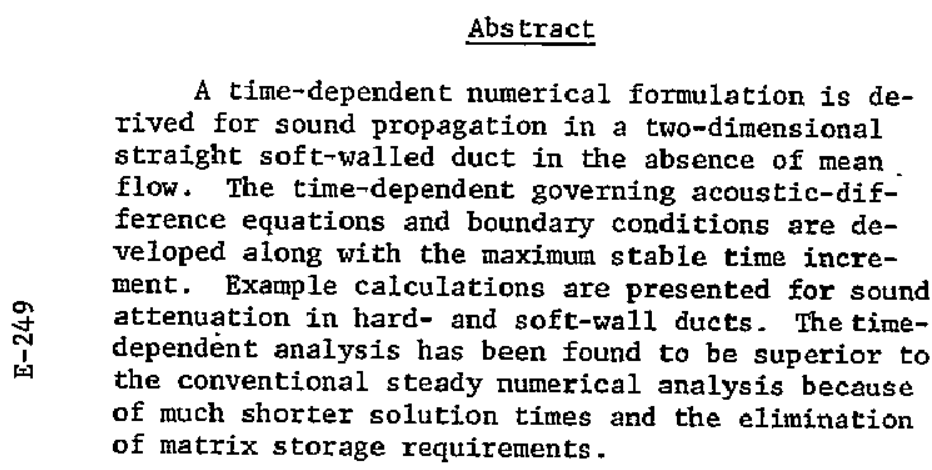

Nomenc1ature

$a_{\text {m }}$ cell coefficient

b cell coefficient

$c_{m}$ cell coefficient

$c_{0}^{*}$ ambient speed of sound, $\mathrm{m} / \mathrm{s}$

d cell coefficient

$e_{m}$ cell coefficient

$\mathrm{I}^{*}$ frequency, $\mathrm{Hz}$

fin cell coefficient

$\mathrm{g}_{\mathrm{m}}$ cell coefficient

$\mathrm{H}^{*}$ height of duct, $\mathrm{m}$

I number of axial grid points

i $\sqrt{-1}$

$\mathbf{J}$ number of transverse grid points

$L^{*}$ Iength of duct, $m$

n transverse mode number

$P \quad$ time-dependent acoustic pressure, $P * / \rho_{0}^{*} c^{* 2}$

P spatially dependent acoustic pressure

$P_{S}$ spatially dependent solution of Helmholtz equation

$\mathrm{T}^{*}$ period, $1 / \mathrm{f}^{*}$, sec

$t$ dimensionless time, $t^{*} / T^{*}$

$\Delta t \quad$ time step

I axial acoustic velocity, $u^{*} / \mathrm{c}_{\mathrm{o}}^{*}$

$\mathrm{v}$ transverse acoustic velocity, $\mathrm{v}^{*} / \mathrm{c}_{0}^{*}$

$x$ axial coordinate, $x^{*} / H^{*}$

$\Delta \mathrm{x} \quad$ axial grid spacing

y dimensionless transverse coordinate, $\mathrm{y}^{*} / \mathrm{H}^{*}$

$\Delta y \quad$ transverse grid spacing

*Aerospace Engineer.

AIAA Paper No, 80-0098.

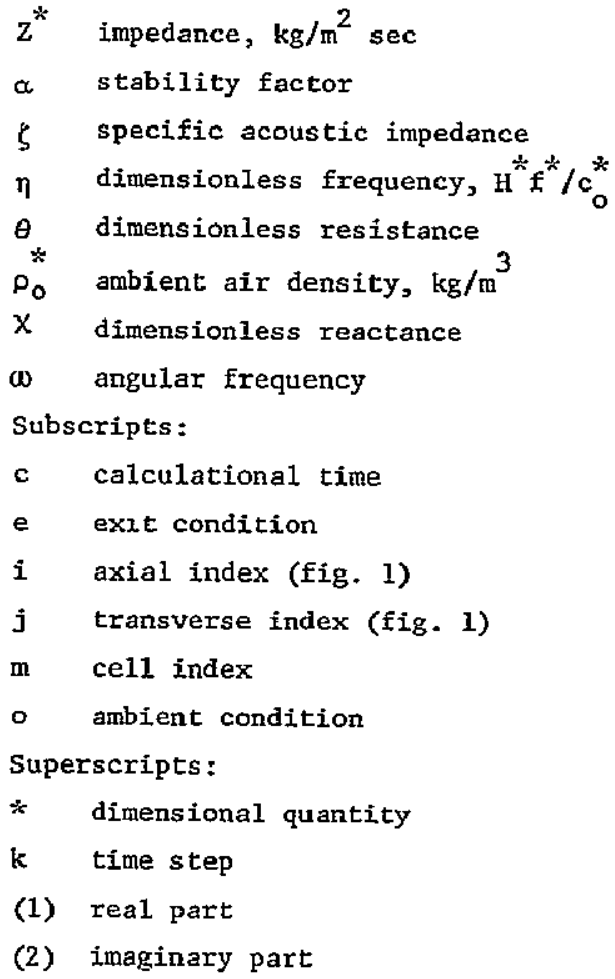

\section{Introduction}

Both finite-difference and finite-element numerical techniques (refs. 1 to 27 ) have been deve1oped to study sound propagation with axial variations in Mach number, wall impedance, and duct geometry as might be encountered in a typical turbojet engine. Generally, the numerical solutions have been limited to low-frequency sound and short ducts, because many grid points or elements were required to resolve the axial wavelength of the sound. As shown in reference 1 (eq. 77) for plane wave propagation, the number of grid points or elements is proportional to the sound frequency and duct length, and inversely proportional to one minus the Mach number (ref. 2, fig. 6). This later dependence severely limsts the application of numerical techniques for high Mach number inlets.

Customarily, the pressure and acoustic velocities are assumed to be simple harmonic functions of time; thus, the governing linearized gas-dynamic equations (ref. 28, p. 5) become independent of time. The matrices associated with the numerical solution to the time independent equations must be solved exactly using such methods as Gauss elimination. Iteration techniques are unstable. As a result, large arrays of matrix elements must be stored which tax the storage capacity of even the largest computer. In unpublished work at Lewis using reference 29 as well as the work of Quinn (ref. 23, p. 3) the matrix has been modified to allow iteration techniques; unfortunately the convergence is too slow to be of any practical value. Other approaches, such as in reference 30 , might still offer iterative possibilities. 
Some special techniques have been developed to overcome the above mentioned difficulties. As shown in references 3 and 10 , the wave envelope numerical technique can reduce the required number of grid points by an order of magnitude. In reference 20 , this technique was used to optimize multielement liners of long lengths at high frequencies. At the present time, this technique has been applied only to the simple cases of no flow and plug flow. A numerical spatial marching technique was also developed in references 15 and 18 . Compared to the standard finite-difference or finite-element boundary value approaches, the numerical marching technique is orders of magnitude shorter in computational time and required computer storage. The marching technique is limited to high frequencies and when reflections are small.

As an alternative to the previously developed steady state theories, a time-dependent numerical technique is developed herein for noise propagation in a two-dimensional soft-wall duct in the absence of mean flow. Advantageously, matrix storage requirements are significantly reduced in the timedependent analysis. The analysis begins with a noise source radiating into an initially quiescent duct. This explicit method calculates stepwise in real time to obtain the transient as well as the "steady" state solution of the acoustic field. The total time required for the analysis to calculate the "steady" state acoustic field will determine the usefulness of the time-dependent technique.

Time-dependent numerical techniques have been applied extensively to both one-dimensional sound propagation (ref. 31 , p. 258 ), two-dimensional vibration problems (ref. 32, p. 452), and the more general problem of compressible fluid flow (ref. 33). References 31, 34, and 35 discuss in detail the stability of numerical solutions to the wave equation. Herein, these techniques will be extended to include soft-wall impedance boundary conditions which would be encountered in inlets and exhaust ducts of turbofan engines.

In the present paper, the governing acousticdifference equation and the appropriate boundary conditions associated with time-dependent propagation are presented. Next, the von Neumann method is used to develop the relationship between sound frequency and grid spacing to determine the maximum stable time increment. Immediately following the mathematical development, numerical solutions are presented for one- and two-dimensional hard- and soft-wall ducts. The results are compared with the corresponding steady analytical results. Finally, the time required to perform both the time-dependent and steady analyses are compared for increasing number of grid points.

\section{Governing Equations and Boundary Conditions}

The propagation of sound in a two-dimensional rectangular duct, as shown in figure 1 , is described by the linearized continuity and momentum equations and the appropriate impedance boundary conditions.

\section{Gontinuity and Momentum}

The linearized equations for mass and momentum conservation can be written (ref. 28, p. 5) for a Gartesian coordinate system in the following dimensionless form:

$$
\begin{gathered}
\frac{\partial P}{\partial t}=-\frac{1}{\eta} \frac{\partial u}{\partial x}-\frac{1}{\eta} \frac{\partial v}{\partial y} \\
\frac{\partial u}{\partial t}=-\frac{1}{\eta} \frac{\partial P}{\partial x} \\
\frac{\partial v}{\partial t}=-\frac{1}{\eta} \frac{\partial P}{\partial y}
\end{gathered}
$$

These and other symbols are defined in the nomenclature. The dimensionless frequency $\eta$ is defined as

$$
\eta=\left(\frac{\mathrm{H}^{*}}{2 \pi}\right) \frac{\omega^{*}}{\mathrm{c}_{0}^{*}}=\frac{\mathrm{H}^{*} \mathrm{E}^{*}}{\mathrm{c}_{\mathrm{O}}^{*}}
$$

The asterisks denote dimensional quantities.

The foregoing dimensionless equations apply to the scaled Cartesian coordinate system in which the height ranges between 0 and $I$ and the dimensionless length ranges between 0 and $\mathrm{L}^{*} / \mathrm{H}^{*}$.

\section{Wave Equation}

Equations ( 1 to 3 ) now are combined to yield the dimensionless wave equation

$$
\eta^{2} \frac{\partial^{2} P}{\partial t^{2}}=\frac{\partial^{2} P}{\partial x^{2}}+\frac{\partial^{2} P}{\partial y^{2}}
$$

Equation (5) in difference form will be solved to determine the pressure in the duct.

\section{WaIl Boundary Condition}

The boundary condition at the surface of a sound absorbent soft-wall duct can be expressed in terms of a specific acoustic impedance defined as

$$
\zeta=\frac{z^{*}}{\rho c_{0}^{*}}=\frac{p}{v}
$$

Substituting equation (6) into equation (3) yie1ds

$$
\frac{\partial \mathrm{P}}{\partial \mathrm{y}}=-\frac{\eta}{\zeta} \frac{\partial \mathrm{P}}{\partial \mathrm{t}}+\frac{\eta \mathrm{P}}{\xi^{2}} \frac{\partial \zeta}{\partial t}
$$

In the example problems to be considered, the impedance will be asstumed constant with time. Therefore, equation (7) reduces to

$$
\frac{\partial P}{\partial y}=-\frac{\eta}{\xi} \frac{\partial P}{\partial t}
$$

At the lower wall, the sign on $\zeta$ is changed to account for the vector nature of $v$. It is also convenient to express the specific acoustic impedance in terms of resistance $\theta$ and reactance $X$ as

$$
\zeta=\theta+i x
$$

\section{Entrance Condition}

The boundary condition at the source plane $P(o, y, t)$ can be of any general form with both trans* verse variations in pressure and multiple frequency content. However, the numerical technique will be compared later to previous solutions in which the pressure and acoustic velocities were assumed to be 
plane waves at the entrance and to vary as $\mathrm{e}^{i 0 \times t *}$ or in dimensionaless form as $e^{i 2 \pi t}$. Therefore, the source boundary condition used here in

$$
P(0, y, t)=e^{i \cdot 2 \pi t}
$$

\section{Exit Impedance}

In a manner similar to the wall impedance, the axial impedance at the duct exit can be defined as

$$
\zeta_{\mathrm{e}}=\frac{\mathrm{P}\left(\mathrm{L}^{*} / \mathrm{H}^{*}, \mathrm{y}, \mathrm{t}\right)}{\mathrm{u}\left(\mathrm{L}^{*} / \mathrm{H}^{*}, \mathrm{y}, \mathrm{t}\right)}
$$

For the plane wave propagation to be considered herein, $\zeta_{e}$ is taken as 1 , which is exact for plane wave propagation in an infinite hard-wall duct. A1so choosing $\zeta_{e}$ to be 1 has lead to close agreement between numerical and analytical results for plane wave propagation into a soft-wall duct (refs. $I$ and 3). More general values for the exit impedance can be found in references 7,15 (eq. (B4)), 16 , or 18 (fig. 7).

\section{Initial Condition}

- For times equal to or less than zero, the duct is assumed quiescent, that is, the acoustic pressure and velocities are taken to be zero. For times greater than zero, the application of the noise source (eq. (10)) will drive the pressures in the duct.

\section{Complex Notation}

Because of the introduction of complex notation for the noise source and wall impedances, a11 the dependent variables are complex. The superscript (1) will represent the real term while (2) will represent the imaginary term;

$$
P=P^{(1)}+i p^{(2)}
$$

A similar notation applies to the acoustic velocities.

\section{Difference Equations}

Instead of a continuous solution for pressure in space and time, the finite-difference approximations will determine the pressure at isolated grid points in space as shown in figure 1 and at discrete time steps $\Delta t$. Starting from the known initial conditions at $t=0$ and the boundary conditions, the finite-difference algorithm will march-out the solution to later times.

\section{Gentral Region (Cell \#1)}

Away from the duct boundaries, in cell \#1 of figure 1 , the second derivatives in the wave equation (eq. (5)) can be xepresented by the usual central differences in time and space (ref. 34, p. 99)

$$
\begin{array}{r}
\eta^{2}\left(\frac{P_{i, j}^{k+1}-2 P_{i, j}^{k}+P_{i, j}^{k-1}}{\Delta t^{2}}\right)=\left(\frac{p_{i+1, j}^{k}-2 P_{i, j}^{k}+P_{i-1, j}^{k}}{\Delta x^{2}}\right) \\
+\left(\frac{P_{i, j+1}^{k}-2 P_{i, j}^{k}+P_{i, j-1}^{k}}{\Delta y^{2}}\right)
\end{array}
$$

where $i$ and $j$ denote the space indices, $k$ the time index, and $\Delta x, \Delta y$, and $\Delta t$ are the space and time mesh spacing, respectively. All spacings are assumed constant. The time is defined as

$$
t^{k+1}=t^{k}+\Delta t=(k+I) \Delta t
$$

Solving equation (13) for the pressure $P_{i, j}^{k+1}$ yields

$$
\begin{aligned}
P_{i, j}^{k+1}=2 P_{i, j}^{k}-P_{i, j}^{k-1} & +\frac{\alpha}{\left[1+\left(\frac{\Delta y}{\Delta x}\right)^{2}\right]}\left\{\left(\frac{\Delta y}{\Delta x}\right)^{2} P_{i-1, j}^{k}\right. \\
& +P_{i, j-1}^{k}-2\left[1+\left(\frac{\Delta y}{\Delta x}\right)^{2}\right] P_{i, j}^{k} \\
& \left.+P_{i, j+1}^{k}+\left(\frac{\Delta y}{\Delta x}\right)^{2} P_{i+1, j}^{k}\right\}
\end{aligned}
$$

where $\alpha$ is defined as

$$
\alpha=\frac{\Delta t^{2}}{\eta^{2} \Delta y^{2}}\left[1+\left(\frac{\Delta y}{\Delta x}\right)^{2}\right]
$$

Equation (15) is an algorithm which permits marching out solutions from known values of pressures at times associated with $k$ and $k-1$. The procedure is explicit since all the past values of $\mathrm{p}^{k}$ are known as the new values of $k+1$ are computed. For the special case at $t=0$, the values of the pressure associated with the $k-1$ value are zero from the assumed initial condition. The parameter $a$ was introduced into equation (15) because it will play an important role in determining the stability (error growth) of equation (15) in this explicit iteration scheme.

\section{Boundary Condition (Cel1s \#2 to \#6)}

The expressions for the difference equations at the waIl boundary are complicated by the impedance condition and the change in geometry of cells \#2 through \#6 in figure 1 . The governing difference equations can be developed by an integration process in which the wave equation (eq. (5)) is integrated over the area of the cells and time:

$$
\int_{t-\Delta t / 2}^{t+\Delta t / 2} \iint_{\substack{\text { Cel1 } \\ \text { area }}}\left(\eta^{2} \frac{\partial^{2} p}{\partial t^{2}}-\frac{\partial^{2} p}{\partial x^{2}}-\frac{\partial^{2} p}{\partial y^{2}}\right) d x d y d t=0
$$

The spatial integration over the cell area is fully documented in reference 10, appendix D. In equation (17) of this paper, the spatial integration method of reference 10 has been extended to include time. An illustration of the application of equation (17) to cell $\# 2$ is given in the appendix of this paper. 
The finite-difference approximation for the various cells shown in figure 1 are expressed in terms of the cell coefficients $a_{m}$ through $g_{m}$ :

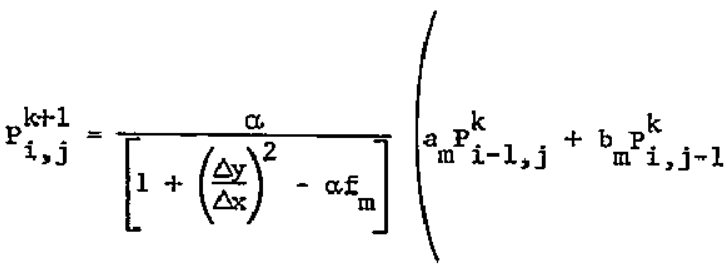

$$
\begin{aligned}
& +\left\{c_{m}+\frac{2\left[1+\left(\frac{\Delta y}{\Delta x}\right)^{2}\right]}{\alpha}\right\} P_{i, j}^{k}+d_{m} p_{i, j-1}^{k} \\
& +e_{m} \mathrm{P}_{i+1, j}^{k} \mid-\frac{\left[1+\left(\frac{\Delta y}{\Delta x}\right)^{2}-\alpha g_{m}\right]}{\left[1+\left(\frac{\Delta y}{\Delta x}\right)^{2}-\alpha \dot{F}_{m}\right]} \mathrm{P}_{i, j}^{k-1}
\end{aligned}
$$

The subscript m denotes the cell number. These coefficients are listed in table $I$.

\section{Spatial Mesh Size}

The mesh spacings $\Delta x$ and $\Delta y$ must be restricted to small values to reduce the truncation error. To resolve the oscillatory nature of the pressure the required number of grid points in the axial direction suggested in reference 1 was

$$
I \geq 12 \eta \frac{L^{*}}{\mathrm{H}^{*}}
$$

No requirement for the size of transverse spacing $\triangle y$ was given in reference 1 other than the number of transverse grid points be increased until convergence is achieved.

In the rectangular duct shown in figure 1 , propagating transverse acoustic pressure modes (cos nTy) can exist in the duct (ref. 15, eq. (B2)) when

$$
n \leq 2 \eta
$$

To resolve all the propagating modes, the number of grid points in the transverse direction, $J$, suggested here is

$$
\mathbf{J} \geq 12 \eta
$$

Equation (19) in conjunction with equation (21) would lead to equal axial and transverse mesh spacing which generally minimizes the truncation error (ref. 33, p. 288)

\section{Stability}

In the explicit time marching approach used here, round-off errors can grow in an unbounded fashion and destroy the solution if the time increment $\Delta t$ is taken too large. The von Neuman method is often used to study the stability of the difference approximations to the wave equation. Application of the von Neuman method (ref. 34, p. 104) to equation (13) requires that $\alpha$ in equation (16) be less than 1; which limits the time increment

$$
\Delta t \leqq \frac{\Delta y}{\sqrt{1+\left(\frac{\Delta y}{\Delta x}\right)^{2}}}
$$

The derivation in reference 34 was for only one space dimension; however, the extension of the von Neuman method to two space dimension is relatively easy. When the time step satisfies equation (22), a condition of linear instability exists ( $r$ ef. 34, p. 106), which guarantees that a propagating acoustic mode can travel undiminished in a hard-wall duct (see discussion, ref. 18, p. 302).

\section{Steady State Pressures}

In the sample problems to be presented in the next section, the time-dependent restults will be compared to the results of the steady harmonic solutions of reference 10 . The purpose of the section is to show the rationale for constructing a steady state solution from the time-dependent results.

\section{Steady Harmonic Solutjon}

The steady harmonic pressure $p_{f}(x, y)$ is defined as a solution to equation (5) when the pressure is assumed to be a simple harmonic function of time :

$$
P(x, y, t)=P_{S}(x, y) e^{i 2 \pi t}
$$

Substituting equation (23) into equation (5), the wave equation takes the form of the classic Helmholtz equation

$$
\frac{\partial^{2} p_{s}}{\partial x^{2}}+\frac{\partial^{2} p_{s}}{\partial y^{2}}+(2 \pi \eta)^{2} p_{s}=0
$$

In this case, where the source is a simple harmonic function of time, $p$ represents the Fouxier transform of $P(x, y, t)$ (ref. 28, p. 11). The boundary conditions can also be modified by equation (23) as shown in reference 10 .

For a semiinfinite duct (or an equivalent finite duct with $\rho^{*} c^{*}$ exit Impedance) with plane wave propagation, and hard walls, the solution for $p_{s}$ is

$$
\mathrm{P}_{\mathrm{S}}=\mathrm{e}^{-i 2 \pi \eta \mathrm{x}}
$$

In the next section, a transient solution to this problem will be compared to equation (25).

\section{Transient Solution}

Reca11, at the start of the numerical calculation, the acoustic pressures and velocities were assumed zero throughout the duct and a pressure source begins a harmonic oscillation at $x=0$ for $t \geq 0$. For the special case of plane wave propagation in a hard-wall, seminfinite duct, the analytical solution to the wave equation (eq. (5)) is (ref. 36, p. 305) 


$$
P(x, t)= \begin{cases}0 & 0<t<\eta x \\ e^{-i 2 \pi \eta x} e^{i 2 \pi t} & t>\eta x\end{cases}
$$

The pressure $p(x, y)$ is now defined by dividding the instantaneous pressure $P(x, y, t)$ by $e^{i 2 \pi t}$ to obtain

$$
p(x, y)=\frac{P(x, y, t)}{e^{i 2 \pi t}}
$$

Using this definition, equations (26) and (27) become

$$
P(x)= \begin{cases}0 & 0<t<\eta x \\ e^{-i 2 \pi \eta x} & t>\eta x\end{cases}
$$

Consequently, the transient solution (eq. (30)) for the steady harmonic pressure equals the Fourier transform solution (eq. (25)) when

$$
t>\eta x
$$

In terms of real variables, equation (31) can be written as

$$
t^{*}>\frac{x^{*}}{c_{0}^{*}}
$$

The transient time $t^{*}$ represents the time for the wave to travel down to the end of the duct, $x^{*}=I^{*}$. Therefore, for the special case of one-dimensional plane wave propagation, the initial transient will pass when equation (3I) holds.

Since it may be desirable to integrate the wave with time to obtain a rms (root mean squared) pressure, the transient calculations will be continued into the steady domain for one period of oscillation before the Fourier pressure $p$ is calculated. Therefore, in this paper,

$$
t_{c}=\frac{\eta L^{*}}{H^{*}}+I
$$

and

$$
p(x, y)=\frac{P(x, y, t)}{e^{i 2 \pi t} c}
$$

For more complicated problems, such as with higherorder acoustic pressure modes or where reflections are important, $t$ should be increased in successive steps to chêck for convergence.

\section{Sample Calculations}

In two sample problems to follow, the timedependent results will be compared to the results of the steady harmonic solutions of reference 10 .

\section{Hard-Wa11 Duct}

Numerical and analytical values of the pressure $p(x, y)$ are computed for the case of a hard- wa11 duct for plane wave propagation with $\zeta=1$ exit impedance (equivalent to seminfinite duct). The calculation was made with a length to height ratio $\left(\mathrm{L}^{*} / \mathrm{H}^{*}\right)$ of 1 and a dimensionless frequency $\eta$ of 1. The analytical and numerical values of the acoustic pressure profiles along the duct are shown in figure 2. As seen in figure 2, agreement between analytical and the numerical theory is good.

\section{Soft-Wall Duct}

As another example of the time-dependent analysis, the pressure distributions are computed for the case of plane wave propagation with a $\zeta=1$ exit impedance and a wall with impedance values of $0.16-$ 10.34. The calculation was made with a length-toheight ratio of 0.5 and a dimensionless frequency of 0.6. The results of the time-dependent analysis along with the results of the solution of the equivalent steady state Helmhomtz equation are displayed in figure 3. The numerical results for the steady spatial solution $p_{s}(x, y)$ are tabulated in appendix $F$ of reference 10 . As seen in figure 3 , again the steady and time-dependent analyses are in good agreement.

\section{Grid Point Variations}

Figure 4 shows the effect of increasing the number of grid points on the computational time of the time-dependent approach for the hard-wall duct associated with figure 2. Roughly, as seen in figure 4 , the computational time is proportional to the number of grid points used. This is a considerable advantage over the steady technique in which the computational time more nearly increases with the square of the total grid points.

Figure 5 shows a comparis on of the computational times for the steady and time-dependent problems associated with the hard-wall duct shown in figure 2 . As seen in this figure, the time-dependent analysis is considerably faster than the steady analysis. The value of $J$ (grid points in $y$-direction) was restricted to 20 because of practical limitations on the size of the matrix which could be effectively handled in the steady analysis. When $\mathbf{J}$ was in creased to 50 with 50 axial grid points, the steady analysis required 5500 seconds as compared to less than 20 seconds for the time-dependent analysis. This large increase in the steady state solution time results because of the manner in which the general matrix was partitioned (ref. 10, p. 14). In this case, the storage and computational times are proportional to the total number of transverse grid points squared $(J)^{2}$.

Finally, these calculations were performed on the Univac 1100 comptiter. Faster computers can be expected to significantly reduce these calculational times.

\section{Conclusions}

With the possible exception of the wave envelope technique (ref. 10) or the spatial marching technique (ref. 18), the numerical time-dependent method of analysis represents a significant advance over previous steady numerical theories. By eliminating large matrix storage requirements, numerical calculations of high sound frequencies are now possible. Also, because matrix manipulation is not required, the time-dependent approach is simpler to 
program and debug. Al though flow has not been considered herein, the extension to the more general flow situation appears to be"straightforward.

\section{APPENDIX}

\section{Finite-Difference Equations and Coefficients}

The derivation of the difference equation for the various cells starts with equation (17), which is rewritten here as

$\int_{t-\Delta t / 2}^{t+\Delta t / 2} \int_{-}^{+}\left(\eta^{2} \frac{\partial^{2} p}{\partial t^{2}}-\frac{\partial^{2} P}{\partial x^{2}}-\frac{\partial^{2} p}{\partial y^{2}}\right) d x d y d t=0$

where the plus sign ( $t$ ) in the upper limit of integration means to evaluate the parameters along either the upper or right-hand boundary of the integration cell, shown in figure 1 by the dashed lines, while the negative sign (-) applies to either the lower or left-hand boundary of the integration cell, depending on whether $x$ or $y$ is considered. The $(+)$ and $(-)$ notation was used since the spatial integration limsts will vary from cell to ce11. For cell \#2 for example, in the $x$ intetral, (+) is represented by $x+\Delta x / 2$ and $(-)$ by $x-\Delta x / 2$, while in the $y$ integral, $(+)$ is represented by 1 and $(-)$ by $1-\Delta y / 2$.

The pressure $\mathrm{P}$ can be assumed constant over the cell area and likewise the second derivative in time can also be assumed constant over the entire cell area. Therefore, moving the spatial derivatives to the right side of equation (Al) gives

$$
\begin{aligned}
& \eta^{2} \int_{t-\Delta t / 2}^{t+\Delta t / 2} \frac{\partial^{2} P_{i, j}}{\partial t^{2}} d t\left(\int_{-}^{+} \int_{-}^{+} d x d y\right)=\int_{t-\Delta t / 2}^{t+\Delta t / 2} \\
& x \int_{-}^{+} \int_{-}^{+}\left(\frac{\partial^{2} p}{\partial x^{2}}+\frac{\partial^{2} P}{\partial y^{2}}\right) d x d y d t
\end{aligned}
$$

but

$$
\begin{gathered}
\left.\int_{t-\Delta t / 2}^{t+\Delta t / 2} \frac{\partial^{2} p}{\partial t^{2} d t=\frac{\partial p}{\partial t}}\right|_{t-\Delta t / 2} ^{t+\Delta t / 2}=\left(\frac{P_{i, j}^{k+1}-P_{i, j}^{k}}{\Delta t}\right) \\
-\left(\frac{P_{ \pm, j}^{k}-P_{i, j}^{k-1}}{\Delta t}\right)=\frac{P_{i, j}^{k+1}-2 P_{i, j}^{k}+P_{i, j}^{k-1}}{\Delta t}
\end{gathered}
$$

Therefore, equation (A2) can be written as

$$
P_{i, j}^{k+1}=2 P_{i, j}^{k}-P_{i, j}^{k-1}+\frac{\alpha}{\left[1+\left(\frac{\Delta y}{\Delta x}\right)^{2}\right]} \Psi_{m}
$$

where

$$
\begin{aligned}
\Psi_{m}=\frac{\Delta y^{2}}{\Delta t} \int_{t-\Delta t / 2}^{t+\Delta t / 2}\left[\int_{-}^{+}\left(\left.\frac{\partial p}{\partial x}\right|^{+}-\frac{\partial p}{\partial x} \mid\right) d y\right. \\
\left.+\int_{-}^{+}\left(\left.\frac{\partial p}{\partial y}\right|^{+}-\frac{\partial P}{\partial y} \mid\right) d x\right] d t
\end{aligned}
$$

The parameter $\Psi_{m}$ can now be evaluated for each cell (labeled $\mathrm{m}$ ) in figure 1 . The procedure for each cell is given in detail in reference 10, appen$\operatorname{dix}$ D. To illustrate how the time integration is coupled to the space integration, the derivation for ce11 \#2 will now be presented.

For cell \#2,

$$
\int_{-}^{+} \int_{-}^{t} d y d x=\frac{\Delta y \Delta x}{2}
$$

$$
\begin{gathered}
\left.\frac{\partial P}{\partial x}\right|^{+}-\left.\frac{\partial P}{\partial x}\right|_{-}=\left(\frac{P_{i+1, j}^{k}-P_{i, j}^{k}}{\Delta x}\right)-\left(\frac{P_{i, j}^{k}-P_{i-1, j}^{k}}{\Delta x}\right) \\
=\frac{P_{i+1, j}^{k}-2 P_{i, j}^{k}+P_{i-1, j}^{k}}{\Delta x}
\end{gathered}
$$

$$
\left.\frac{\partial P}{\partial y}\right|^{+}-\left.\frac{\partial p}{\partial y}\right|_{-}=-\frac{\eta}{\zeta} \frac{\partial P_{i, j}}{\partial t}-\left(\frac{P_{i, j}^{k}-P_{i, j-1}^{k}}{\Delta y}\right)
$$

The expression for $\left.\frac{\partial p}{\partial y}\right|^{+}$in equation (A8) is the wall boundary condition as given by equation ( 8 ) in the body of this report. Substituting equations $(A 6),(A 7)$, and (A8) into equation (AS) yie1ds

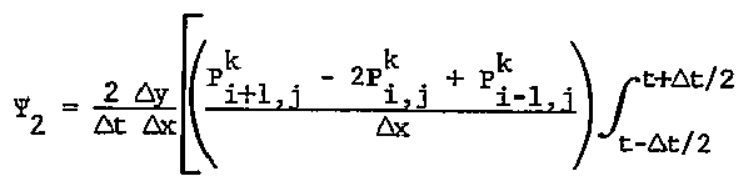

$$
\begin{aligned}
& x d t \int_{-}^{+} d y-\frac{n}{\zeta} \int_{t-\Delta t / 2}^{t+\Delta t / 2} \frac{\partial P_{i, j}}{\partial t} d t \int_{-}^{+} \\
& \left.x d x-\left(p_{i, j}^{k}-p_{i, j-1}^{k}\right) \int_{t-\Delta t / 2}^{t+\Delta t / 2} d t \int_{-}^{+} d x\right]
\end{aligned}
$$
noting
Equation (A9) can be further simplified by 


$$
\begin{aligned}
& \int_{-}^{+} d y=\frac{\Delta y}{2} \\
& \int_{-}^{+} d x=\Delta x \\
& \int_{t-\Delta t / 2}^{t+\Delta t / 2} d t=\Delta t \\
& \int_{t-\Delta t / 2}^{t+\Delta t / 2} \frac{\partial P}{\partial t} d t=\left.P_{i, j}\right|_{t-\Delta t / 2} ^{t+\Delta t / 2}=\left(\frac{P_{i, j}^{k+1}+P_{i, j}^{k}}{2}\right) \\
& -\left(\frac{P_{i, j}^{k}+P_{i, j}^{k-I}}{2}\right)=\frac{P_{i, j}^{k+1}-P_{i, j}^{k-1}}{2}
\end{aligned}
$$

Substituting equations (A10) to (A13) into equation (A9) yields

$$
\begin{aligned}
\Psi_{2} & =\left(\frac{\Delta y}{\Delta x}\right)^{2} P_{i-1, j}^{k}+2 P_{i, j-1}^{k}-2\left[1+\left(\frac{\Delta y}{\Delta x}\right)^{2}\right] P_{i, j}^{k} \\
& +\left(\frac{\Delta y}{\Delta x}\right)^{2} P_{i+1, j}^{k}-\frac{\eta}{\zeta} \frac{\Delta y}{\Delta t} P^{k+1}+\frac{\eta}{\zeta} \frac{\Delta y}{\Delta t} P_{i, j}^{k-1}
\end{aligned}
$$

which contain the coefficients that appear in table I for cell \#2. The coefficients for the other cells are found in a similar manner.

\section{References}

1. Baumeister, K. J. and Bittner, E. C., "Numerical Simulation of Noise Propagation in Jet Engine Ducts," NASA TN $\mathrm{D}-7339,1973$.

2. Baumeister, K. J. and Rice, E. J., "A Difference Theory for Noise Propagation in an Acoustically Lined Duct with Mean Flow," Aeroacoustics: Jet and Combustion Noise; Duct Acoustics, Progress in Astronautics and Aeronautics, Vol. 37, American Institute of Aeronautics and Astronautics, New York, 1975, p. 435-453.

3. Baumeister, K. J., "Analysis of Sound Propagation in Ducts Using the Wave Envelope Concept," NASA TN D-7719, 1974.

4. Quinn, D. W., "A Finite Difference Method for Computing Sound Propagation in Nonuniform Ducts," AIAA Paper 75-130, Jan. 1975.

5. Baumeister, K. J., "Wave Envelope Analysis of Sound Propagation in Ducts with Variable Axial Empedance," Aeroacoustics: Duct Acoustics, Fan Noise and Control Rotor Noise, I. R. Schwartz, H. T. Nagamatsu, and W. Strahle, eds: Progress in Astronautics and Aeronautics, Vol. 44, American Institute of Aeronautics and Astronautics, New York, 1976, pp. 451-474.

6. Quinn, D. W., "Attenuation of Sound Associated with a Plane Wave in a Multisectional Duct,"
Aeroacoustics: Duct Acoustics, Fan Noise and Control Rotor Noise, I. R. Schwartz, H. T. Nagamatsu, and W. Strahle, eds., Progress in Astronautics and Aeronautics, Vol. 44, American Institute of Aeronautics and Astronautics, New York, 1976, PP. 331-345.

7. Sigman, R. K., Majjigi, R. K., and Zimn, B. T., "Determination of Turbofan Inlet Acoustics Using Finite Elements," AIAA Journal, Vol. 16, Nov. 1978, pp. 1139-1145.

8. Abrahamson, A. I., "A Finite Element Algorithm for Sound Propagation in Axisymmetric Ducts Containing Compressible Mean Flow," Wyle Labs., Inc., Hampton, VA, June 1977. (NASA (RR-145209)

9. Craggs, A., "A Finite Method for Model1ing Disdipative Mufflers with a Locally Reactive Linning," Journal of Sound and Vibration, Vol. 54, Sep. 1977, PP. 285-296.

10. Baumeister, K. J., "Finite-Difference Theory for Sound Propagation in a Lined Duct with Uniform Flow Using the Wave Envelope Concept," NASA TP-1001, 1977.

11. Kagawa, Y., Xamabuchi, T., and Mori, A., "Finite Element Simulation of an Axisymmetric Acoustic Transmission System with a Sound Absorbing Wa11," Journal of Sound and Vibration, Vol. 53, Aug. 1977, pp. 357-374.

12. Eversman, W., AstIey, R. J., and Thanh, V. P., "Transmission in Nonuniform Ducts - A Comparative Evaluation of Finite Element and Weighted Residuals Computational Schemes," AIAA Paper 77-1299, Oct. 1977.

13. Watson, H. R., "A Finite Element Analysis of Sound Propagation in a Rectang ular Duct of Finite Length with Peripherally Variable Liners," AIAA Paper 77-1300, Oct. 1977.

14. Abrahamson, A. L., "A Finite Element Algorithm for Sound Propagation in Axisymmetric Ducts Containing Compressible Mean Flow, "AIAA Paper 77-1301, 0ct. 1977.

15. Baumeister, K. J., "Numerical Spatial Marching Techniques for Estimating Duct At tenuation and Source Pressure Profiles," 95th Meeting Acoustical Soclety of America, Providence, Rhode Island, May 16-19, 1978. (Also NASA TM78857,1978 )

16. Tag, I. A. and Lumsdaine, E., "An Efficient Finite Element Technique for Sound Propagation. in Axisymmetric Hard Wall Ducts Carrying High Subsonic Mach Number FIows, AIAA Paper 78-1154, July 1978.

17. Astley, R. J. and Eversman, W., "A Finite Element Method for Transmission in Non-Uniform Ducts without Flow: Comparison with the Method of Weighted Residuals, "Journal of Sound and Vibration, Vo1. 57, Apr. 1978, pp. $367-388$.

18. Baumeister, K. J., "Numerical Spatial Marching Techniques in Duct Acoustics," Journal of the Acoustical Society of America, Vol. 65, Feb. 1979, pp. 297-306. 
19. Majjigi, R. K., "Application of Finite Element Techniques in Predicting the Acoustic Properties of Turbofan Inlets," Ph.D. Thesis, Georgia Institute of Technology, Atlanta, GA, 1979.

20. Baumeister, K. J., "0tpimized Multisectioned Acoustic Liners," AIAA Paper 79-0182, Jan. 1979.

21. Majjigi, R. K., Sigman, R. K., and Zinn, B. T. "Wave Propagation in Ducts Using the Finite Element Method," AIAA Paper 79-0659, Mar. 1979.

22. Astley, R. J. and Eversman, W., "The Application of Finite Element Techniques to Acoustic Transmission in Iined Ducts with Flow," AIAA Paper 79-0660, Mar. 1979.

23. Quinn, D. W., "A Finite Element Method for Contputing Sound Propagation in Ducts Containing Flow," AIAA Paper 79-0661, Mar. 1979.

24. Abrahamson, A. L., "Acoustic Duct Liner Optimization Using Finite Elements," AIAA Paper 79-0662, Mar. 1979.

25. Tag, I. A. and Akin, J. E., "Finite Element Solution of Sound Propagation in a Variable Area Duct," AIAA Paper 79-0663, Mar. 1979.

26. Lester, H. C. and Parrott, T. L., "Application of Finite Element Method for Computing Grazing Incidence Wave Structure in an Impedance Tube: Comparison with Experiment," AIAA Paper 79-0664, Mar. 1979.

27. Baumeister, K. J. and Majjigi, R. K., "Applications of Velocity Potential Function to Acoustic Duct Propagation and Radiation from Inlets
Using Finite-Element Theory," AIAA Paper 79-0680, Mar. 1979.

28. Goldstein, M. E., Aeroacoustics, McGraw-Hill, New York, 1976.

29. Beaubien, M. J. and WesIer, A., "Iterative, Finite Difference Solution of Interior Eigenvalues and Eigenfunctions of Laplace, $s$ Operator," Computer Journal, Vol. 14, Aug. 1971, pp. $263-269$.

30. Browne, B. T. and Lawrenson, P. J., Numerical Solution of an Elliptic Boundary-Value Problem in the Complex Variable," Institute of Mathematics and its Applications Journal, Vol. 17, 1976, Pp, 311-327.

31. Richtmyer, R. D. and Morton, K. W., Difference Methods for Initial-Value Problems, 2nd ed., Interscience, New York, 1967.

32. Gerald, C. F., Applied Numerical Analysis, 2nd ed., Addison-Wesley, Reading, MA, 1978.

33. Roache, P. J., Computational Flujid Dynamics, Hermosa, Albuquerque, NM, 1972.

34. Clark, M. and Hansen, K. F., Numerical Methods of Reactor Analysis, Academic Press, New York, 1964 .

35. Hildebrand, F. B., Methods of Applied Mathematics, Printice-Hall, Englewood Cliffs, NJ, 1952.

36. Budak, B. M., Samarskii, A. A., and Tikhonov, A. N., A Collection of Problems on Nathematical Physics, Pergamon, (Oxford), 1964.

TABLE I. - COEFFICIENTS IN DIFFERENCE EQUATIONS

\begin{tabular}{|c|c|c|c|c|c|c|c|}
\hline \multirow{2}{*}{$\begin{array}{l}\text { Ce11 } \\
\text { index, } \\
\text { m }\end{array}$} & \multicolumn{7}{|c|}{ Difference elements* } \\
\hline & $a_{m}$ & $b_{\text {ml }}$ & $c_{m}$ & $d_{m}$ & $e_{m}$ & $\mathrm{E}_{\mathrm{m}}$ & $\mathrm{g}_{\mathrm{m}}$ \\
\hline 1 & $\left(\frac{\Delta y}{\Delta x}\right)^{2}$ & 1 & $-2\left[1+\left(\frac{\Delta y}{\Delta x}\right)^{2}\right]$ & 1 & $\left(\frac{\Delta y}{\Delta x}\right)^{2}$ & 0 & 0 \\
\hline 2 & $a_{1}$ & 2 & $c_{1}$ & 0 & $-e_{1}$ & $-\frac{\eta}{\zeta} \frac{\Delta y}{\Delta t}$ & $\frac{\eta}{\zeta} \frac{\Delta y}{\Delta t}$ \\
\hline 3 & $a_{1}$ & 0 & $c_{1}$ & 2 & $e_{1}$ & $-\frac{\eta}{\zeta} \frac{\Delta y}{\Delta t}$ & $\frac{\eta}{\zeta} \frac{\Delta y}{\Delta t}$ \\
\hline 4 & $2 a_{1}$ & 1 & $c_{1}$ & 1 & 0 & $-\frac{\eta \Delta y^{2}}{\xi_{e} \Delta t \Delta x}$ & $\frac{\eta \Delta y^{2}}{\zeta_{e} \Delta t \Delta x}$ \\
\hline 5 & $2 a_{1}$ & 2 & $c_{1}$ & 0 & 0 & $\mathrm{f}_{2}+\mathrm{f}_{4}$ & $g_{2}+g_{4}$ \\
\hline 6 & $2 a_{1}$ & 0 & $c_{1}$ & 2 & 0 & $f_{3}+f_{4}$ & $g_{3}+g_{4}$ \\
\hline
\end{tabular}


TIME-DEPENDENT NUMERICAL RESULTS

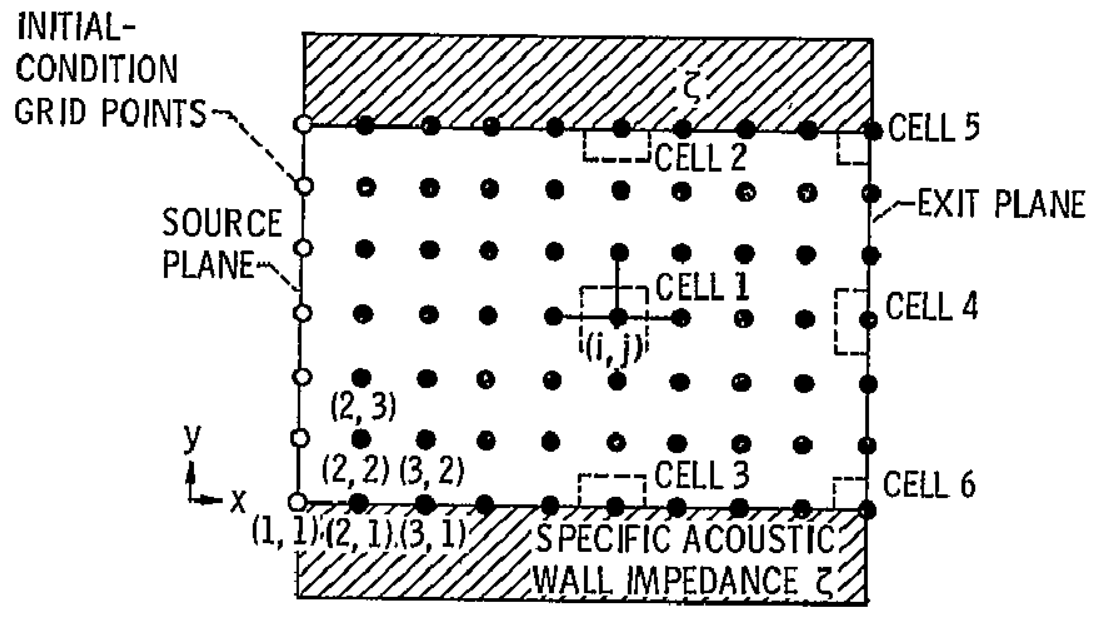

Figure 1. - Grid-point representation of two-dimensional flow duct.
REAL PART, $p^{(l)}$

IMAGINARY PART, $p^{(2)}$

EXACT ANALYSIS

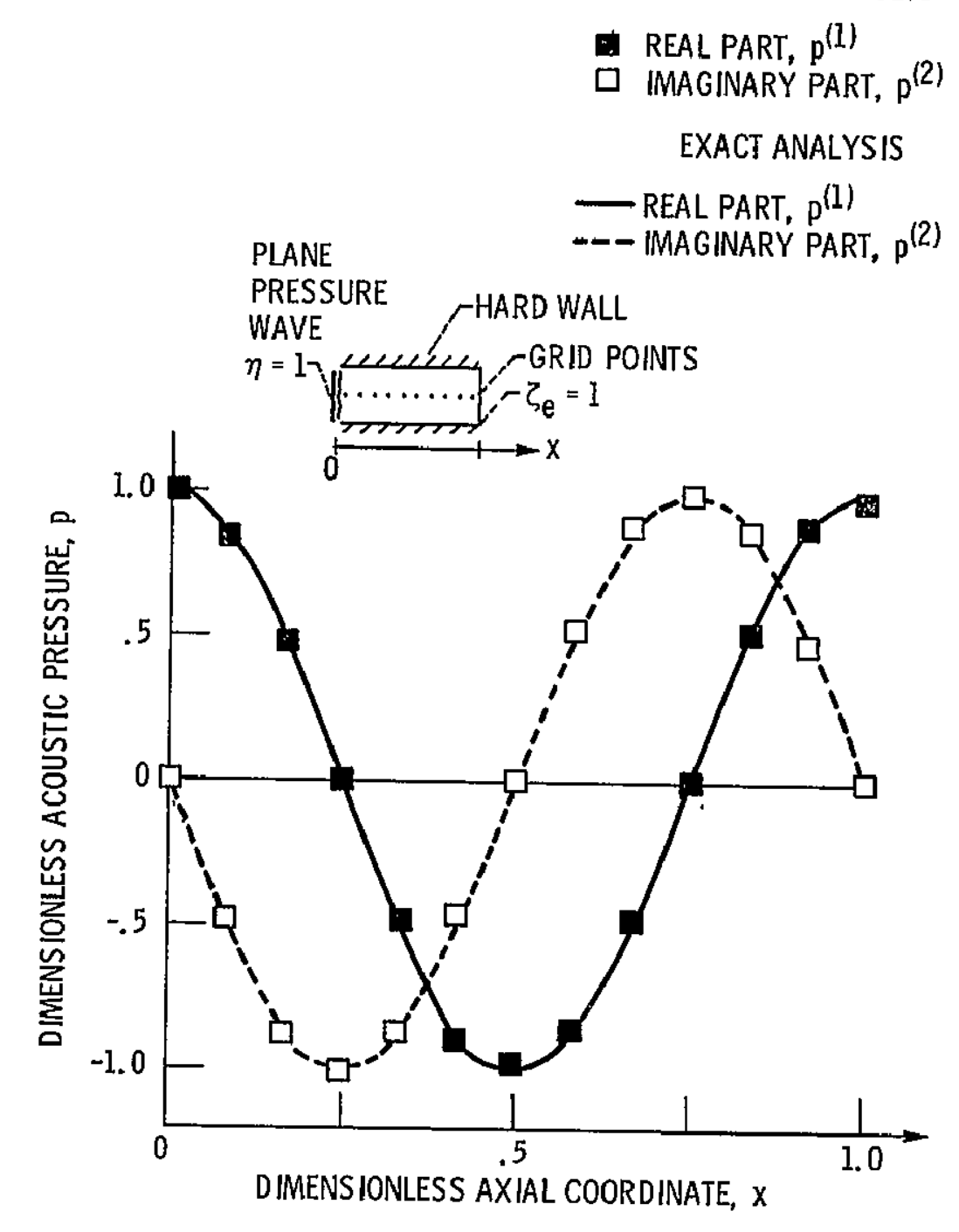

Figure 2. - Analytical and numerical pressure profiles for one-dimensional plane wave sound propagation in a hard

wall duct for $\eta=1$ and $L * / H^{*}=1$. 


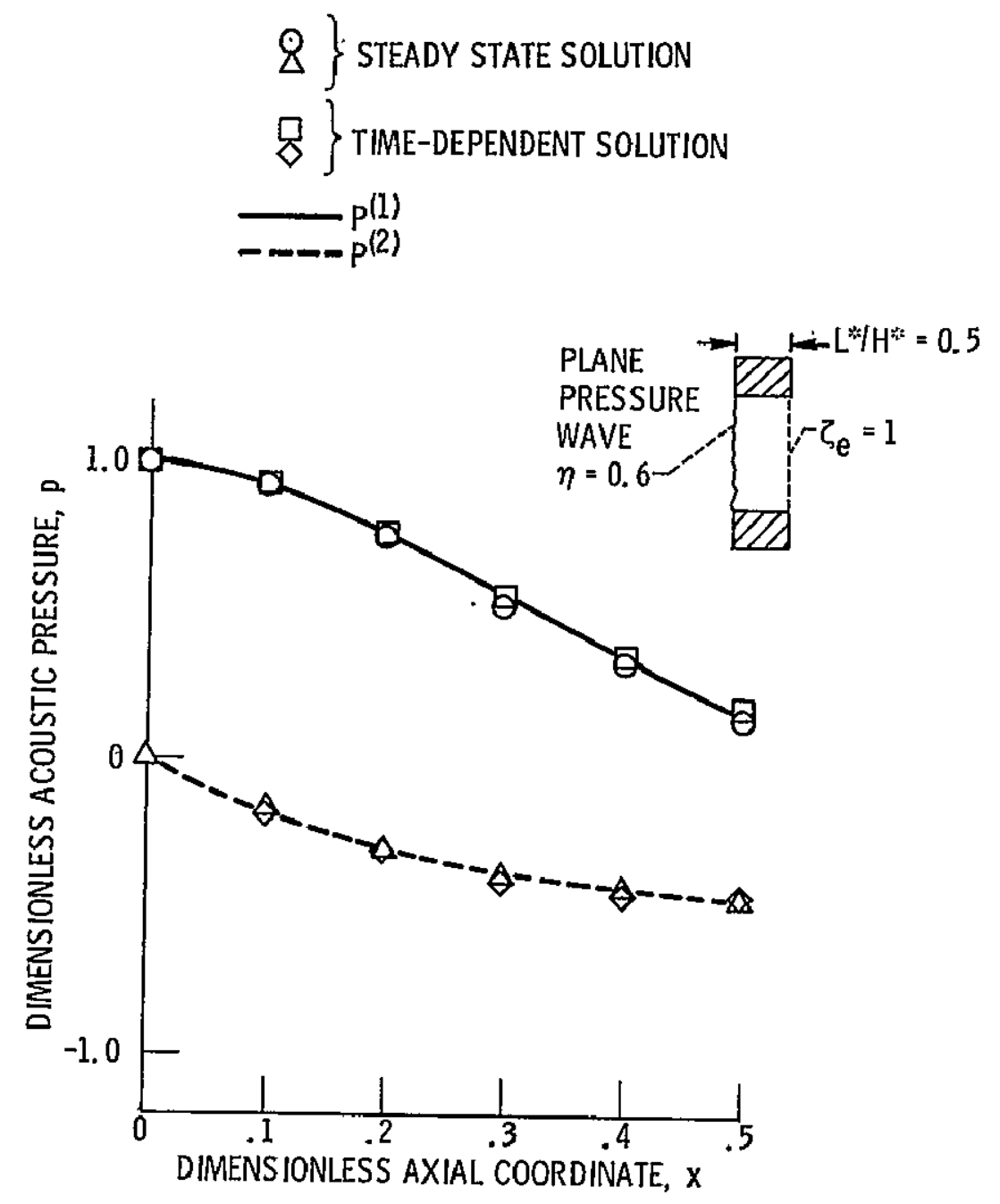

Figure 3. - Pressure profiles on the duct axis for incident plane wave sound propagation in a soft wall duct $(\eta=0.6$, $\left.L^{*} / H^{*}=0.5, \zeta_{W}=0.16-i 0.34\right)$.

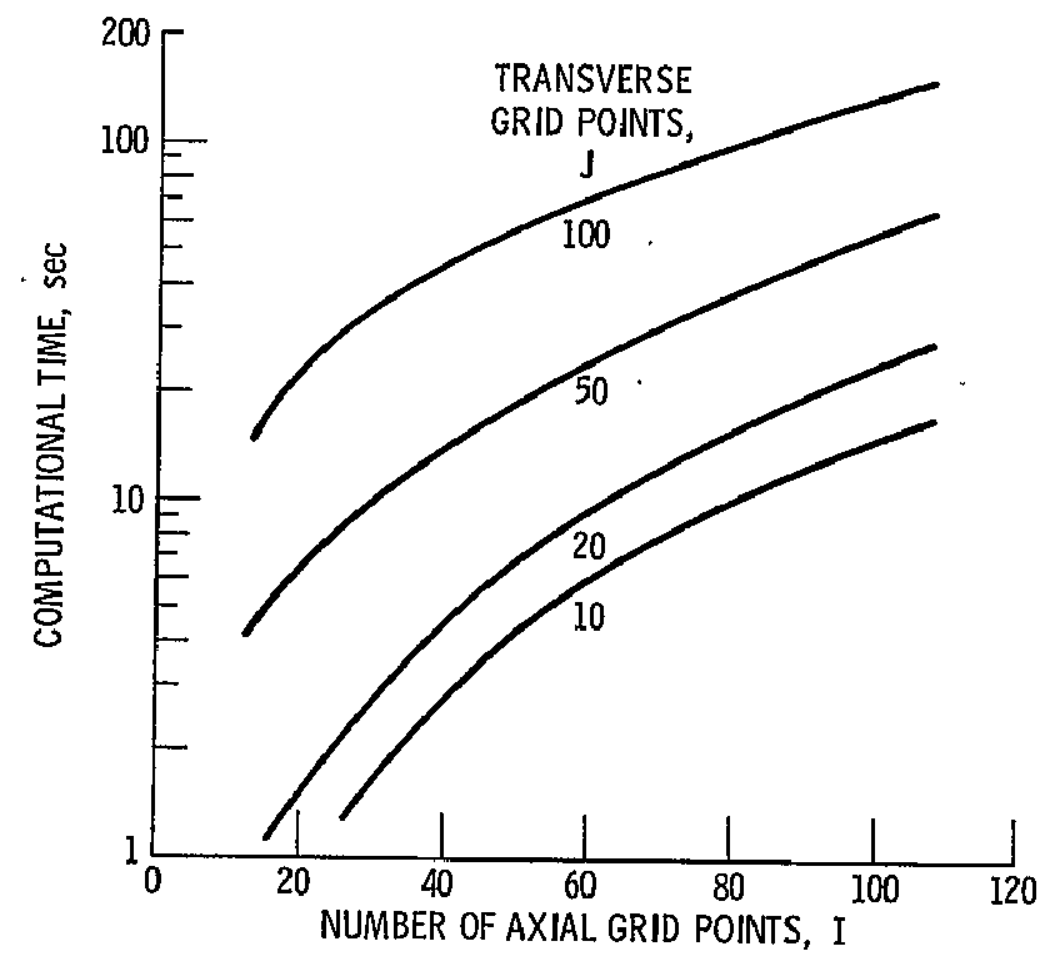

Figure 4. - Effect of increasing number of grid points on calculational time of transient solution for plane wave propagation in a hard wall duct $\left(\eta=1, L * / H^{*}=1\right)$. 


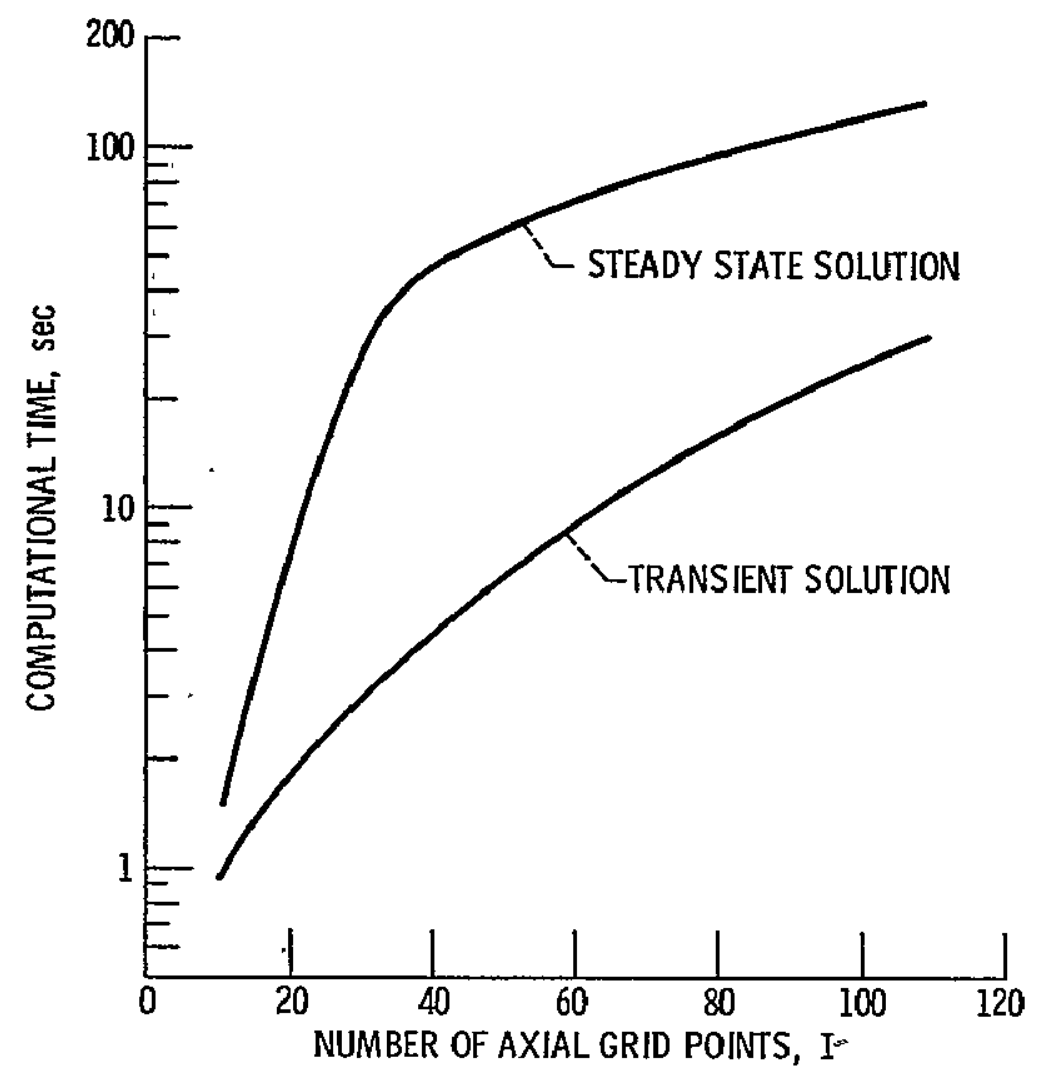

Figure 5. - Comparison of transient and steady state calculations for plane wave propagation in a hard wall duct $\left(\eta=1, L^{*} / H^{* *}=1, J=20\right)$. 


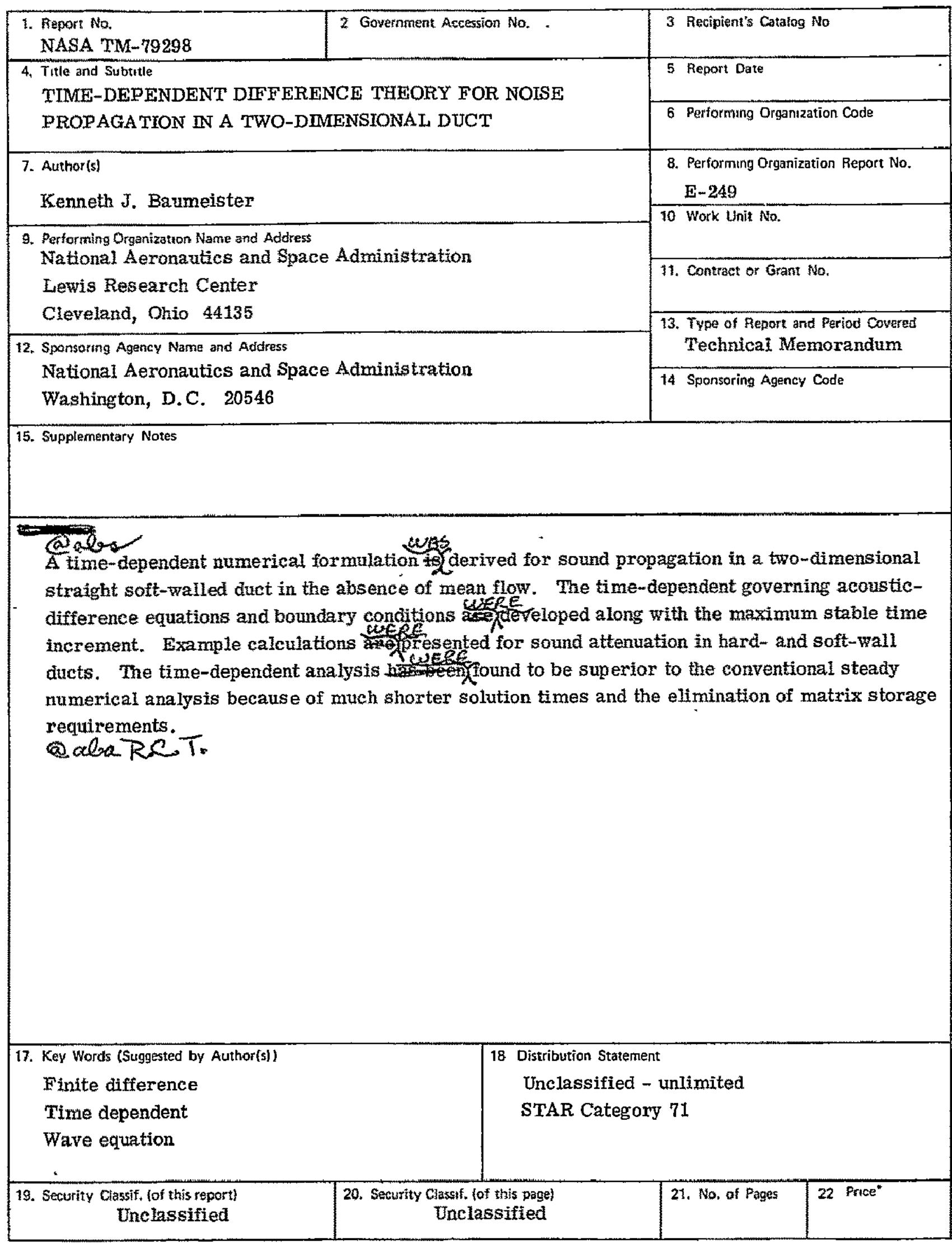

* For sale by the National Technical Informaton Service, Snungfield, Vugtnia 22161 
National Aeronautics and Space Administration

SPECIAL FOURTH CLASS MAIL BOOK

Washington, D.C.

20546

\section{Official Business}

Penalty for Private Use, $\$ 300$

Postage and Fees Paid

National Aeronautics and Space Administration

NASA-451

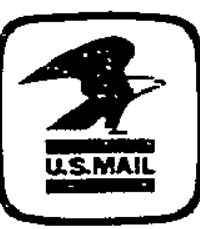

